\title{
Structure of the Plasmodium falciparum Triosephosphate Isomerase-Phosphoglycolate Complex in Two Crystal Forms: Characterization of Catalytic Loop Open and Closed Conformations in the Ligand-Bound State
}

\author{
S. Parthasarathy, ${ }^{\S}$ G. Ravindra, ${ }^{\S}$ Hemalatha Balaram, ${ }^{\|}$P. Balaram, ${ }^{\S}$ and M. R. N. Murthy*, $\S$ \\ Molecular Biophysics Unit, Indian Institute of Science, Bangalore 560 012, India, and Molecular Biology and Genetics Unit, \\ Jawaharlal Nehru Center for Advanced Scientific Research, Jakkur, Bangalore 560 064, India
}

Received March 6, 2002; Revised Manuscript Received August 29, 2002

\begin{abstract}
Triosephosphate isomerase (TIM) has been the subject of many structural and mechanistic studies. At position 96, there is a highly conserved Ser residue, which is proximal to the catalytic site. Thus far, no specific role has been ascribed to this residue. Plasmodium falciparum TIM (PfTIM), a fully catalytically active enzyme, is unique in possessing a Phe residue at position 96. The structure of PfTIM complexed to phosphoglycolate (PG), a transition state analogue, has been determined in an effort to probe the effects of the mutation at residue 96 on the nature of inhibitor-enzyme interactions and the orientation of the critical catalytic loop (loop 6, residues 166-176) in TIM. Crystal structures of PfTIM complexed to phosphoglycolate in orthorhombic $\left(P 2_{1} 2_{1} 2_{1}\right)$ and monoclinic $(C 2)$ forms were determined and refined at resolutions of 2.8 and $1.9 \AA$, respectively. The $P 2_{1} 2_{1} 2_{1}$ form contains two dimers in the asymmetric unit. In the $C 2$ form, the molecular and crystal 2 -fold axes are coincident, leading to a monomer in the asymmetric unit. The catalytic loop adopts the open state in the $P 2{ }_{2} 2_{1} 2_{1}$ form and the closed conformation in the $C 2$ crystal. The open conformation of the loop in the $P 2_{1} 2_{1} 2_{1}$ form appears to be a consequence of the Ser to Phe mutation at residue 96. The steric clash between Phe96 and Ile172 probably impedes loop closure in PfTIM-ligand complexes. The PfTIM-PG complex is the first example of a TIM-ligand complex being observed in both loop open and closed forms. In the C2 form (loop closed), Phe96 and Leu167 adopt alternative conformations that are different from the ones observed in the open form, permitting loop closure. These structures provide strong support for the view that loop closure is not essential for ligand binding and that dynamic loop movement may occur in both free and ligandbound forms of the enzyme.
\end{abstract}

Malaria is a major global public health problem. Plasmodium falciparum $(\mathrm{Pf})^{1}$ is the most virulent among the malarial parasites. The development of drug resistance in Pf strains has stimulated considerable interest in the search for new antimalarial drugs and drug targets. As part of a program to develop metabolic enzymes as potential drug targets (1), we have determined the crystal structure of Pf triosephosphate isomerase (PfTIM) at $2.2 \AA$ resolution (2). The focus on glycolytic enzymes in the malarial parasite results from the observation that in the asexual stage of the parasite in the

\footnotetext{
$\dagger$ The work reported here is supported by grants from the Council of Scientific and Industrial Research (CSIR) and the Department of Science and Technology (DST) of the Government of India. S.P. is the recipient of a CSIR research fellowship.

The loop closed and open structures are PDB entries $1 \mathrm{LYZ}$ and $1 \mathrm{LYO}$, respectively.

* To whom correspondence should be addressed. Telephone: 9180-3092458. Fax: 91-80-3600683 or 91-80-3600535. E-mail: mrn@ mbu.iisc.ernet.in.

$\S$ Indian Institute of Science.

"Jawaharlal Nehru Center for Advanced Scientific Research.

${ }^{1}$ Abbreviations: TIM, triosephosphate isomerase; Pf, Plasmodium falciparum; PfTIM, P. falciparum triosephosphate isomerase; DHAP, dihydroxyacetone phosphate; D-GAP, D-glyceraldehyde 3-phosphate; 3PG, 3-phosphoglycerate; G3P, glycerol 3-phosphate; PG, phosphoglycolate; Tryp, trypanosomal; TrypTIM, Trypanosoma brucei triosephosphate isomerase.
}

human erythrocytes, the energy requirements of the organism are almost exclusively met by glycolysis (3).

For the development of an enzyme as a drug target, it is essential to probe the key structural differences between host and pathogen enzymes, which can then in principle be used to develop selective inhibitors. A notable feature of the PfTIM sequence is the presence of a Phe residue at position 96. In all other TIM sequences determined so far, this residue is Ser [see Figure 1 for a multiple-sequence alignment of all TIMs for which crystal structures have been determined thus far (4-12)]. Most intriguingly, the Ser96Phe mutation is also observed in the sequences of triosephosphate isomerases from Plasmodium berghei, Plasmodium yeoli, Plasmodium knowlesi, Plasmodium chabandi, and Plasmodium vivax. The PfTIM gene with the Ser96Phe mutation has also been identified in the whole genome sequence of $P$. falciparum (www.sanger.ac.uk). Residue 96 is proximal to the active site of the enzyme, which is primarily composed of three residues (Lys12, His95, and Glu165). In an early analysis of yeast TIM, a putative role was ascribed to all the residues proximal to the substrate binding site. However, the role of Ser96 was considered to be a "mystery" (13). The observation that the E165D/S96P and H95N/S96P pseudorevertants of chicken TIM are 20- and 60-fold better 


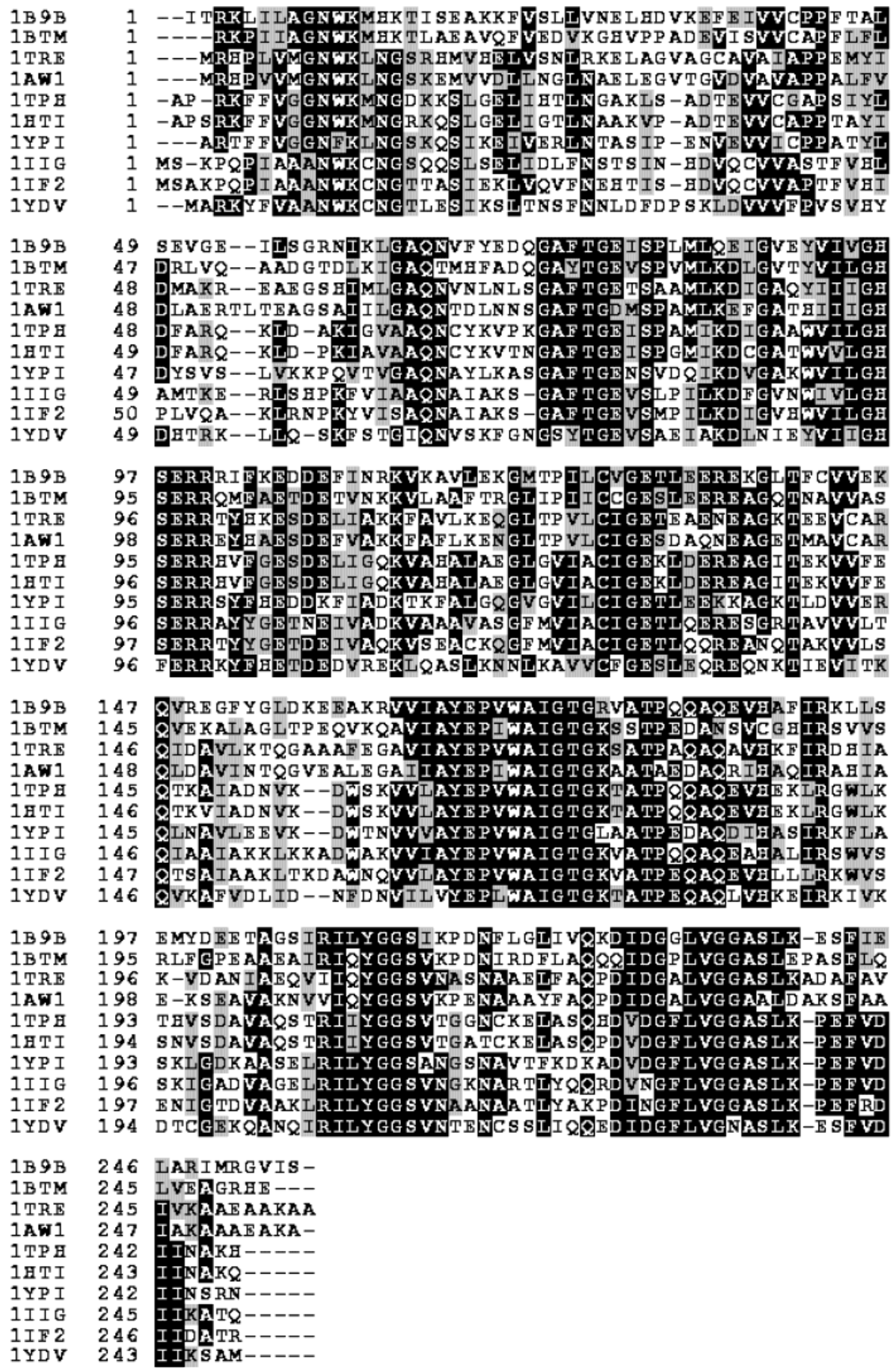

FIGURE 1: Multiple-sequence alignment of triosephosphate isomerases whose crystal structures have been determined. PDB entries 1B9B (Thermotoga maritima; 4), 1BTM (Bacillus stearothermophilus; 5), 1TRE (E. coli; 6), 1AW1 (Vibrio marinus; 7), 1TPH (chicken; 8), 1HTI (human; 9), 1YPI (yeast; 10), 1IIG (T. brucei; 11), 1IF2 (Leishmania mexicana; 12), and 1YDV (P. falciparum; 2).

catalysts than the parent E165D and H95N mutants, respectively, further heightens the mystery about the role of residue 96 (14-16). Interestingly, the S96P mutant of chicken TIM is 20-fold catalytically less efficient than the wild-type enzyme. Crystal structure determination of the S96P mutant at $1.96 \AA$ resolution revealed significant changes of the water structure within the active site cavity (17). Indeed, Alber et al. go so far as to state, "We therefore must keep in mind the possibility that all of our mechanistic deductions about TIM are completely incorrect, since they provide no role for Ser-96. Mutations of this residue into all 19 other amino acids would seem warranted" (13). The natural S96F mutation in PfTIM offers a unique opportunity to further examine the role of this residue in binding and catalysis. PfTIM is fully catalytically competent with $k_{\text {cat }}$ and $K_{\mathrm{m}}$ values of $(2.68 \pm 0.84) \times 10^{5} \mathrm{~min}^{-1}$ and $0.35 \pm 0.16$ with glyceraldehyde 3-phosphate (GAP) as the substrate (18).

TIM has been the subject of intense mechanistic and structural studies over the past three decades. Knowles and Albery suggested in 1977 that TIM is a catalyst, which has been honed to perfection by the "selective pressures of evolution" (19). In 1991 Knowles, concluded that, "if a single message emerges after all the mechanistic and structural scrutiny of TIM, it is one of precision" (20). The crystal structure determinations of TIM from as many as 11 different sources have revealed complete conservation of the $\beta_{8} \alpha_{8}$ 
barrel fold. Structures of complexes of the enzyme with substrate analogues have provided atomic level information about the details of protein-ligand interactions $(11,13,21$, 22).

A notable feature of these complexes is the large movement of a flexible loop (loop 6, residues 166-176) upon ligand binding. Several loop residues, Ile170 and Gly171 in particular, have been implicated in anchoring the substrate at the active site. A loop deletion mutant of chicken TIM in which residues $170-173$ were excised showed a $10^{5}$-fold reduction in catalytic activity (23). Kinetic analysis and isotopic partitioning studies suggest that the loss of activity is a consequence of a "much higher activation barrier for the enolization of the enzyme-bound substrate". The deleted residues of the loop were also implicated in "stabilization of the enediol phosphate intermediate", preventing phosphate elimination and conversion to methylglyoxal (23). Loop 6 has been suggested to move as a rigid body that "breathes on hinges" (24). Solid state NMR studies have shown that the loop movement is an inherent property of the enzyme and the nature of the bound ligand affects only the relative populations of the open and closed conformations (25). Recent solution NMR studies have also provided evidence for the view that loop motion and product release are concerted and might be one of the rate-limiting steps during catalysis (26). NMR studies have also given an estimate of the rate of loop movement in TIM on the order of $10^{4} \mathrm{~s}^{-1}$ (27). Sampson and Knowles suggest that the loop closes after the formation of the Michaelis complex (28). Random mutagenesis experiments have been performed to probe the sequence requirements at the hinge regions of this loop (29, 30). The kinetics are limited by a reduced level of substrate binding or product release rather than the rate of loop movement, which in turn is controlled by its interactions with the rest of the protein.

Interestingly, inspection of the loop 6 sequence of PfTIM revealed that residues $166-176$ are nearly identical to those found in TIM from various other sources (Figure 1). The most striking difference observed between PfTIM and the enzyme from other sources is the presence of Phe at position 96 in place of Ser (2). The proximity of the site of mutation to the active site suggested that steric factors might impede loop closure. However, the high observed catalytic activity of PfTIM (18) appears to be inconsistent with the loop open conformation being the catalytically competent state, since one might have anticipated decomposition of the enediol phosphate intermediate. We, therefore, examined the structure of PfTIM complexed to the transition state analogue, phosphoglycolate. In this paper, two different crystal forms are described, $P 2_{1} 2_{1} 2_{1}$ at $2.8 \AA$ and $C 2$ at $1.9 \AA$. Notably, in the orthorhombic form, the ligand is bound in the "loop open" conformation, whereas in the monoclinic form, the complex adopts a "loop closed" conformation.

\section{MATERIALS AND METHODS}

Purification of PfTIM and Crystallization of the PG Complex. For cloning, overexpression, and purification of PfTIM, we followed procedures established previously (2, 31). Briefly, the gene for PfTIM was cloned into the pTrc 99A vector, known as pARC 1008, and overexpressed in Escherichia coli strain AA200, which has a null mutation in the host TIM gene. The cells were initially grown in 100 $\mathrm{mL}$ of Luria broth for $\sim 6 \mathrm{~h}$ at $37^{\circ} \mathrm{C}$ and transferred to $1 \mathrm{~L}$ of terrific broth. After $4 \mathrm{~h}$, cells were induced using IPTG and growth was continued for a further $8 \mathrm{~h}$. Cells were harvested (centrifuged at $5000 \mathrm{rpm}$ for $20 \mathrm{~min}$ at $4{ }^{\circ} \mathrm{C}$ ), washed with a minimal volume of Tris buffer ( $\mathrm{pH} 7.4)$, and crushed using a French press. Proteins dissolved in the cell lysate were initially precipitated using 70\% (42 wt \%/volume) saturated ammonium sulfate and then by $95 \%$ (63 wt $\% /$ volume) saturation. The resultant ammonium sulfate pellet dissolved in $\sim 5 \mathrm{~mL}$ of cold water was dialyzed extensively against Tris buffer (20 mM, pH 8.0). The protein was further purified using an anion exchange Resource Q (from Pharmacia) column using a FPLC system. A 0 to $0.5 \mathrm{M} \mathrm{NaCl}$ gradient was used for eluting the protein. Alternatively, pure protein could also be obtained by subjecting the dialyzed pellet to two rounds of gel filtration on a Sephadex G-100 column. The final purity of the protein was checked using both SDS-PAGE and ESI-MS. Better crystals, however, were obtained with protein samples purified using a Resource Q column than with a Sephadex G-100 column.

Crystals of the PfTIM-PG complex were obtained using hanging drop setups. The precipitating solution contained 12-25\% PEG6000, PEG4000, PEG3350, or PEG1450 in $100 \mathrm{mM}$ MES (pH 6.5) with DTT, EDTA, and $\mathrm{NaN}_{3}(0.05$ $\mathrm{mM}$ each). A stock protein solution was prepared by dissolving the protein at a concentration of $\sim 15 \mathrm{mg} / \mathrm{mL}$ in Milli Q water with a 50- or 100-fold molar excess of the ligand. Equal volumes of the protein stock and the precipitating solution (total of $6 \mu \mathrm{L}$ ) were placed on a presiliconized cover slip, inverted, and sealed on a well containing $600 \mu \mathrm{L}$ of the precipitating solution. The best crystals were obtained with PEG1450. Crystals of different forms appeared within 1 week. Needlelike orthorhombic $\left(P 2{ }_{1} 2_{1} 2_{1}\right)$ and monoclinic $(C 2)$ crystals appeared under similar conditions in different hanging drops.

Data Collection and Processing. The orthorhombic form, which contained two dimers in the asymmetric unit, diffracted X-rays to $2.8 \AA$ resolution at $100 \mathrm{~K}$ (using an Oxford cryocooler system operated with liquid nitrogen). The monoclinic form, which contained only one subunit in the crystallographic asymmetric unit (due to the alignment of molecular and crystallographic 2-fold axes), diffracted at room temperature to $1.9 \AA$ resolution. Complete X-ray diffraction data on both the crystal forms were collected by screen-less oscillation photography using a MAR300 Image Plate system mounted on a Rigaku RU-200 rotating anode $\mathrm{X}$-ray generator equipped with a $200 \mu \mathrm{m}$ focal cup $(\mathrm{Cu} \mathrm{K} \alpha$ radiation, $\lambda=1.5418 \AA$ ). A single crystal of each form was used to obtain complete data. For the $P 2{ }_{1} 2_{1} 2_{1}$ crystal, 203 frames with an oscillation angle of $1^{\circ}$ or $0.4^{\circ}$ were collected in two different orientations, setting the crystal to detector distance $(D)$ to $185 \mathrm{~mm}$. The crystal was exposed for 1800 $\mathrm{s}$ for an angle of $1^{\circ}$ and $1200 \mathrm{~s}$ for an angle of $0.4^{\circ}$. Each frame was a result of three crystal oscillations. Similarly, for the $C 2$ crystal, 185 frames of $1^{\circ}$ oscillation were collected at two different orientations. $D$ was kept at $120 \mathrm{~mm}$, and each frame was a result of exposure for $900 \mathrm{~s}$ and two oscillations. The data sets were processed using the DENZO/ SCALEPACK suite of programs (32). The statistics listed for $P 2_{1} 2_{1} 2_{1}$ are from the output of the data quality program that determines $R_{\text {meas }}(33)$. The mosaicity of the $C 2$ crystal 
form was not refined in SCALEPACK as this led to the rejection of a large number of reflections. It was optimized by trial and error, exploring around the value of 0.4 , evaluating the results by visual inspection of the agreement between the observed and predicted diffraction patterns in DENZO.

Structure Solution and Refinement. The structure of the unliganded wild-type PfTIM has also been determined and refined in both monoclinic (2) and orthorhombic forms (34). The $C 2$ form of unbound PfTIM contained a dimer in the asymmetric unit (2) in contrast to the complex with PG, which contains a monomer. Molecular replacement (MR) (35) was carried out using the AMoRe (36) suite of programs using the coordinates of wild-type PfTIM as the starting model [PDB entry 1YDV (2)]. The structure of the monoclinic form was determined using a monomer of TIM as the starting model. As expected, MR led to straightforward solutions. The models were further refined with the "mlf (maximum likelihood in amplitudes)" option in CNS_0.4 (37, 38). The data between $20.0 \AA$ and the highest available resolution were used for the refinement using reflections with amplitudes greater than $0.1 \sigma$ and selecting $10.0 \%$ of the data for cross validation $(39,40)$. Anisotropic $B$-scaling and bulk solvent corrections were applied throughout the refinement. In the orthorhombic form, 4-fold noncrystallographic restraints relating the individual subunits of the two dimers in the asymmetric unit were used throughout the refinement. A simulated annealing run incorporating torsion angle dynamics was performed to overcome local minima. Noncrystallographic restraints were not applicable for the refinement of the monoclinic form as it contained only one subunit in the crystallographic asymmetric unit. The models were evaluated and corrected at different stages of refinement using sigmaA-weighted $2 F_{\mathrm{o}}-F_{\mathrm{c}}$ and $F_{\mathrm{o}}-F_{\mathrm{c}}$ maps (41) using the interactive model building programs FRODO and $\mathrm{O}(42,43)$. After water molecules had been included, a few cycles of refinement were carried out. The positions of water molecules in the orthorhombic form are not reliable due to the limited resolution of the data. Table 1 lists the refinement statistics.

Structural Analysis. Structural alignments were carried out using the program ALIGN (44) or the lsq_ex option in O (43). The active site superpositions were done using locally available codes. Ligand interactions and symmetry-related contacts were analyzed using the LIGPLOT (45) and CONTACT programs from the CCP4 [Collaborative Computational Project Number 4 (46)]. Structural validation and rebuilding were done using the programs OOPS (47), PROCHECK (48), WHAT_IF (49), and O (43). Structural illustrations were made using the programs Molscript (50), Bobscript (51), Raster3D (52), and InsightII (53).

Determination of Inhibition Constants. Enzyme activity in the presence and absence of inhibitors was assayed using a coupled enzyme assay (54). The reaction mixture contained $1 \mathrm{mM}$ EDTA, $0.24 \mathrm{mM} \mathrm{NADH}$, and $50 \mu \mathrm{g}$ of $\alpha$-glycerophosphate dehydrogenase (coupling enzyme) in $100 \mathrm{mM}$ triethanolamine buffer ( $\mathrm{pH} 7.5)$. The reaction was followed by measuring the decrease in absorbance at $340 \mathrm{~nm}$ using a Shimadzu UV-210A double-beam spectrophotometer. To initiate the reaction, $10 \mu \mathrm{L}$ of a $18.5 \mathrm{nM}$ stock solution of PfTIM (5 ng) was added to a total volume of $1 \mathrm{~mL}$, which contained variable concentrations of the DL-glyceraldehyde
Table 1: Data Collection and Refinement Statistics of the PfTIM-PG Complex in Orthorhombic and Monoclinic Forms

\begin{tabular}{|c|c|c|}
\hline & orthorhombic & monoclinic \\
\hline $\begin{array}{l}\text { space group } \\
\text { cell parameters }\end{array}$ & $\begin{array}{l}P 2_{1} 2_{1} 2_{1} \\
\begin{array}{l}a=50.821 \AA \\
b=106.771 \AA \\
\quad c=181.291 \AA\end{array}\end{array}$ & $\begin{array}{l}C 2 \\
a=88.242 \AA, \\
b=63.437 \AA \\
c=53.886 \AA \\
\beta=117.741^{\circ},\end{array}$ \\
\hline resolution range $(\AA)$ & $20.0-2.8$ & $20.0-1.9$ \\
\hline no. of reflections & 84748 & 198026 \\
\hline no. of unique reflections & 23550 & 10508 \\
\hline overall completion $(\%)$ & 99.5 & 98.4 \\
\hline multiplicity & 3.6 & 18.8 \\
\hline$R_{\mathrm{symm}}{ }^{a}(\%)$ & 11.5 & 6.8 \\
\hline$R_{\text {free }}$ and $R_{\text {cryst }}{ }^{b}(\%$, final $)$ & 31.1 and 23.2 & 21.1 and 18.0 \\
\hline no. of atoms (protein, water, ligand) & $7828,151,18$ & $1985,173,9$ \\
\hline rmsd for bond lengths $(\AA)$ & 0.008 & 0.006 \\
\hline rmsd for bond angles (deg) & 1.346 & 1.352 \\
\hline mean real space $R$-factor and & 0.045 and 0.049 & 0.042 \\
\hline $\begin{array}{l}\text { correlation coefficient }(\AA \text { and } \mathrm{D})^{c} \\
\text { mean } B \text {-values }(\mathrm{A} \text { and } \mathrm{D})^{c, d}\end{array}$ & 0.955 and 0.950 & 0.959 \\
\hline protein & 13.48 & 21.94 \\
\hline ligand & 26.44 and 34.97 & 19.99 \\
\hline water & not refined & 39.97 \\
\hline overall & not calculated & 23.74 \\
\hline \multicolumn{3}{|c|}{$\begin{array}{l}{ }^{a} R_{\text {symm }}=100 \sum|\langle I\rangle-I| / \sum\langle I\rangle .{ }^{b} R_{\text {cryst }}=100 \sum\left|\left(F_{\text {obs }}-F_{\text {calc }}\right)\right| / \sum\left|F_{\text {obs }}\right| \\
{ }^{c} \text { The parameters are listed for one of the unbound and bound (A and } \\
\mathrm{D} \text {, respectively) subunits. }{ }^{d} B \text {-values correspond to group } B \text {-values for } \\
\text { main chain atoms, side chain atoms, and ligand atoms. }\end{array}$} \\
\hline
\end{tabular}

3-phoshphate as the substrate. The inhibition constant, $K_{\mathrm{i}}$, of the phosphoglycolate (Sigma Chemicals) was obtained by analyzing the effect of the inhibitor on the initial velocity of the conversion of glyceraldehyde 3-phosphate in the concentration range of $0.006-0.1 \mathrm{mM}$. The concentration range used for 3-phosphoglycerate (3PG, Fluka Chemicals) was from 0.3 to $3.6 \mathrm{mM}$. Inhibition constants were obtained from the negative intercept on the $x$-axis of a plot of the apparent $K_{\mathrm{m}}$ versus the inhibitor concentration. The $K_{\mathrm{m}}$ values were derived from the Lineweaver-Burk plots.

\section{RESULTS AND DISCUSSION}

The inhibitor phosphoglycolate (PG) contains one less methylene group than the natural substrate dihydroxyacetone phosphate (DHAP) or D-glyceraldehyde 3-phosphate (DGAP). However, PG has been considered to be a transition state analogue because of its resemblance to the plausible transition state of the isomerization reaction in terms of its structure and charge distribution. Further, PG also binds significantly more tightly to the enzyme than either of the substrates, DHAP or D-GAP (55). Thus far, several TIMPG complexes have been determined which are listed in Table 2. In the study presented here, we have obtained two distinct crystal forms $\left(C 2\right.$ and $\left.P 2_{1} 2_{1} 2_{1}\right)$ of the PfTIM-PG complex, which provide new insights into interactions of the catalytic loop.
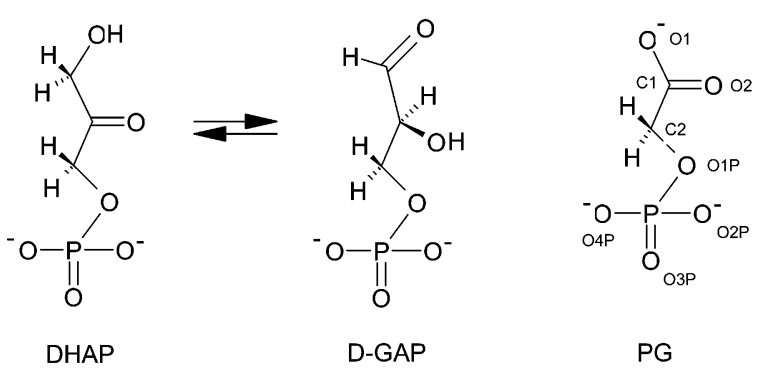
Table 2: Crystal Structures of Phosphoglycolate Complexes of TIM

\begin{tabular}{|c|c|c|c|c|c|}
\hline \multirow[b]{2}{*}{ PDB entry [resolution $(\AA)$, space group] } & \multirow[b]{2}{*}{ source } & \multirow[b]{2}{*}{ asymmetric unit } & \multicolumn{2}{|c|}{ ligand occupancy } & \multirow[b]{2}{*}{ loop conformation (ref) } \\
\hline & & & subunit 1 & subunit 2 & \\
\hline 2 YPI $\left(2.5, P 2_{1}\right)$ & yeast & dimer & bound & bound & closed-closed (55) \\
\hline 1HTI $\left(2.8, P 2{ }_{1} 2_{1} 2_{1}\right)$ & human & dimer & unbound & bound & open-closed (9) \\
\hline $1 \mathrm{BTM}\left(2.8, P 2{ }_{1} 2{ }_{1}\right)$ & B. stearothermophilus & dimer & bound & bound & closed-closed (5) \\
\hline 2BTM $\left(2.4, P 2{ }_{1} 2_{1} 2\right)$ & B. stearothermophilus & dimer & bound & bound & closed-closed (56) \\
\hline 1AW1 $\left(2.7, P 2_{1}\right)$ & V. marinus & four dimers & bound & bound & closed-closed (7) \\
\hline 1QDS $(2.0, C 2)$ & L. mexicana & monomer & bound & - & closed (12) \\
\hline $1 \mathrm{TTI}^{a}(2.4, C 2)$ & T. brucei & dimer & bound & bound & closed-closed (57) \\
\hline $1 \mathrm{ML}^{a}{ }^{a}(2.6, P 3)$ & T. brucei & two trimers & all bound & & closed $(58)$ \\
\hline & P. falciparum & two dimers & bound & bound & open (this study) \\
\hline & P. falciparum & monomer & bound & - & closed (this study) \\
\hline
\end{tabular}

${ }^{a}$ Corresponds to the structure of monoTIM.

\section{Crystal Structures}

Monoclinic C2 Form. In the C2 form of the PfTIM-PG complex, the crystallographic and molecular 2-fold axes are coincident, with the monomer forming the asymmetric unit. Almost all residues are defined well in the electron density map. The map also revealed a shift in electron density for the loop 6 residues, suggesting that the loop might have closed over the active site. Model building essentially involved tracing the closed conformation for loop 6 residues, building the ligand, and adding waters. The final model was refined to $R_{\text {free }}$ and $R_{\text {cryst }}$ values of 21.2 and $18.0 \%$, respectively (Table 1 ).

The final model has good stereochemistry, and only $1.74 \%$ (4 of 230) of non-Gly residues have $\phi$ and $\varphi$ values outside the core region of the Ramachandran plot (59). The outliers are defined according to the method of Kleywegt and Jones (60) as residues lying outside the core areas in which $98 \%$ of all non-Gly residues were found to lie in a sample of 403 PDB structures with a level of sequence identity of $\leq 95 \%$ and determined at resolution $2.0 \AA$ or better. Apart from Lys12 which adopts positive $\phi$ values in all TIM structures determined thus far, Ser211 also shows a positive $\phi$ value, a feature also observed in liganded and loop closed forms of TIM from other sources $(5,61,62)$.

Orthorhombic P $2{ }_{1} 2_{1} 2_{1}$ Form. The $P 2_{1} 2_{1} 2_{1}$ form of the PfTIM-PG complex contains two molecular dimers, leading to four crystallographically independent subunits in the asymmetric unit. The completeness of the intensity data was $99.5 \%$ with an $R_{\text {symm }}$ value of $11.5 \%$ (Table 1 ). Molecular replacement carried out with the unliganded PFTIM dimer as the search model provided the orientation and position of both the dimers. The correlation coefficient and $R$-factors were 28.3 and $51.9 \%$, respectively, after the first dimer had been positioned, and improved to 71.7 and $35.2 \%$, respectively, after the second dimer had been positioned. The structure was refined to $R_{\text {free }}$ and $R_{\text {cryst }}$ values of 31.1 and $23.2 \%$, respectively (Table 1 ). This crystal form of the PfTIM-PG complex yielded significantly lower-quality diffraction data, resulting in correspondingly greater uncertainties in the model stereochemistry in regions where the electron density was poorly defined. The final model has good stereochemistry; 6.4\% (59 of 920) of non-Gly residues have $\phi$ and $\varphi$ values outside the core region of the Ramachandran plot.

The subunits within the crystallographically independent dimers of the orthorhombic form are related by rotations of $179.44^{\circ}$ and $179.88^{\circ}$, respectively. The two dimers of the

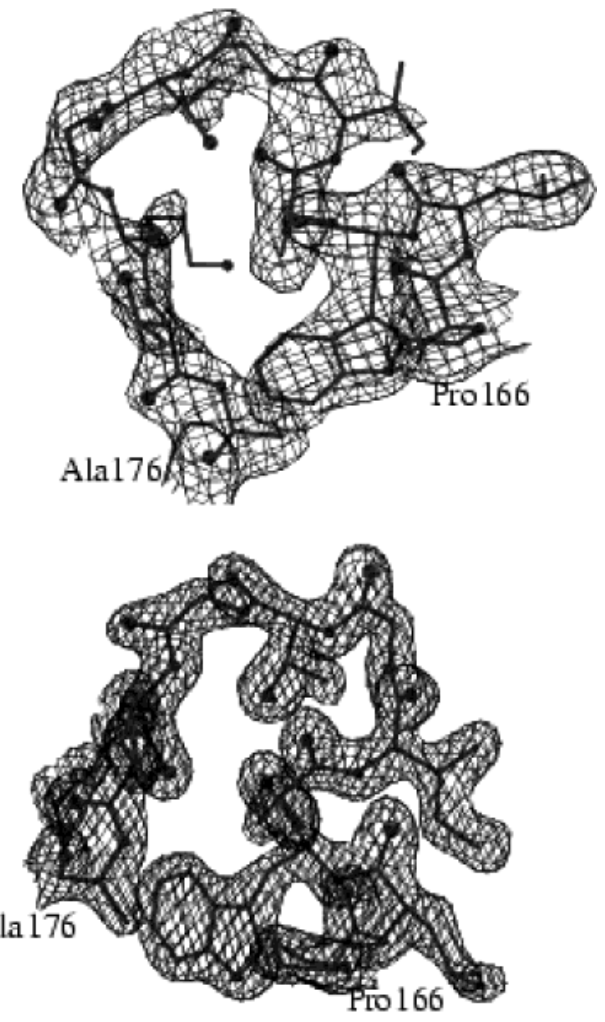

FIGURE 2: Electron density maps $\left(2 m F_{\mathrm{o}}-D F_{\mathrm{c}}\right)$ for loop 6 (residues 166-176) in the bound open (orthorhombic, top panel) and bound closed (monoclinic, bottom panel) forms of the PfTIM-PG complex. Maps are contoured at the $1.0 \sigma$ level.

asymmetric unit are related by a rotation of $103.18^{\circ}$ about an axis that makes angles of $90.24^{\circ}$ and $89.33^{\circ}$ with the 2 -fold axes relating the monomeric units of the two dimers. Thus, the rotation axis relating the two dimers is nearly perpendicular to the plane containing the molecular 2-fold axes relating the subunits of the dimers. Of the four independent subunits, two contain clearly defined electron density corresponding to the bound ligand ( $B$ and $D$ subunits), while in the other two subunits, only partial electron density corresponding to the phosphate group of the bound ligand could be seen.

Conformation of the Catalytic Loop. Figure 2 shows the electron density for the catalytic loop residues (166-176) in the two forms of the PfTIM-PG complex. Loop residues 170-174 are rather poorly defined in four subunits of the orthorhombic form. Further, catalytic loop residues of the ligand-bound B and D subunits are relatively better defined than those of the unbound $\mathrm{A}$ and $\mathrm{C}$ subunits. Electron density 

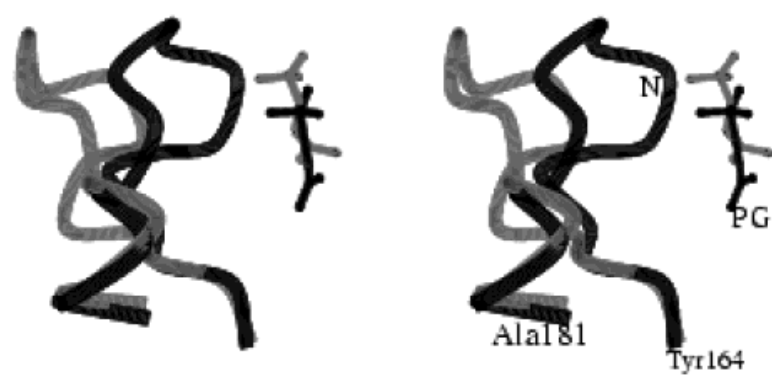

FIGURE 3: Relative positions of the catalytic loops of monoclinic, loop closed (dark) and orthorhombic, loop open forms (light) of the PfTIM-PG complex. Movement of the catalytic loop toward the ligand in the monoclinic form can be seen.

for catalytic loop residues of B subunit is shown in Figure 2 (top panel). As can be seen, side chains of residues Ile170, Thr172, and Lys174 are not defined in the electron density. In contrast, all the residues are clearly defined, including the residues (170-174) in the electron density map in the $C 2$ crystal (Figure 2, bottom panel). Figure 3 shows the relative position of the catalytic loops after superposition of active sites of orthorhombic and monoclinic forms of the PfTIM-PG complex (superposition is done using $\mathrm{C}^{\alpha}$ atoms of Lys12, His95, Glu15, and the residues adjacent to these residues). Movement of the catalytic loop toward the active site can be clearly seen (Figure 3, darker trace). The distances between the $\mathrm{C}^{\alpha}$ atoms of loop 6 residues $(170-176)$ are 3.90 , $6.46,6.86,7.56,4.67,3.73$, and $1.37 \AA$, respectively. These two structures represent the first example of a case where a TIM-ligand complex is observed in both loop open and loop closed conformations.

\section{Protein-Ligand Interactions}

Monoclinic Form (Loop Closed). The monoclinic C2 form of the PfTIM-PG complex contains the bound ligand sequestered in the loop closed conformation of the protein where specific residues on the flexible segment of residues 166-176 (loop 6) make contact with the inhibitor. This is in marked contrast to the structure of the orthorhombic $P 22_{1} 2_{1} 2_{1}$ form where the inhibitor is bound in the loop open conformation (vide infra). Figure 4 (top panel) and Figure 5a show the electron density and LIGPLOT representation of the ligand bound to the active site of the protein, respectively. Table 3 lists the polar interactions using a distance cutoff of $3.8 \AA$ between the ligand and protein atoms. The most important contact made by a residue on the flexible loop with the ligand is the formation of a hydrogen bond between the backbone $\mathrm{NH}$ group of Gly171 and $\mathrm{O} 2 \mathrm{P}$ of $\mathrm{PG}(\mathrm{N}-\mathrm{O}$ distance of $2.73 \AA$ ). This contact, considered to be the fingerprint of the closed conformation of the catalytic loop, is seen in the structures of almost all the TIM-ligand complexes $(13,21,22)$. Apart from this, residues Ala169 and Ile170 from the flexible loop are involved in van der Waals interactions with the ligand.

In contrast to the $P 2_{1} 2_{1} 2_{1}$ form of the PfTIM-PG complex, the catalytic base Glu165 is in direct contact with the carboxylate end of the ligand, which corresponds to the reactive end of the substrate. The carboxylate oxygens, $\mathrm{O} 1$ and $\mathrm{O} 2$, of PG are 3.47 and $2.31 \AA$, respectively, from OE2 of Glu165. The distances between NE2 of His95 and these atoms of PG are 2.69 and $3.58 \AA$, respectively. The $\mathrm{O} 1$ atom of PG is also in direct interaction with NZ of Lys12 at a distance of $2.98 \AA$.
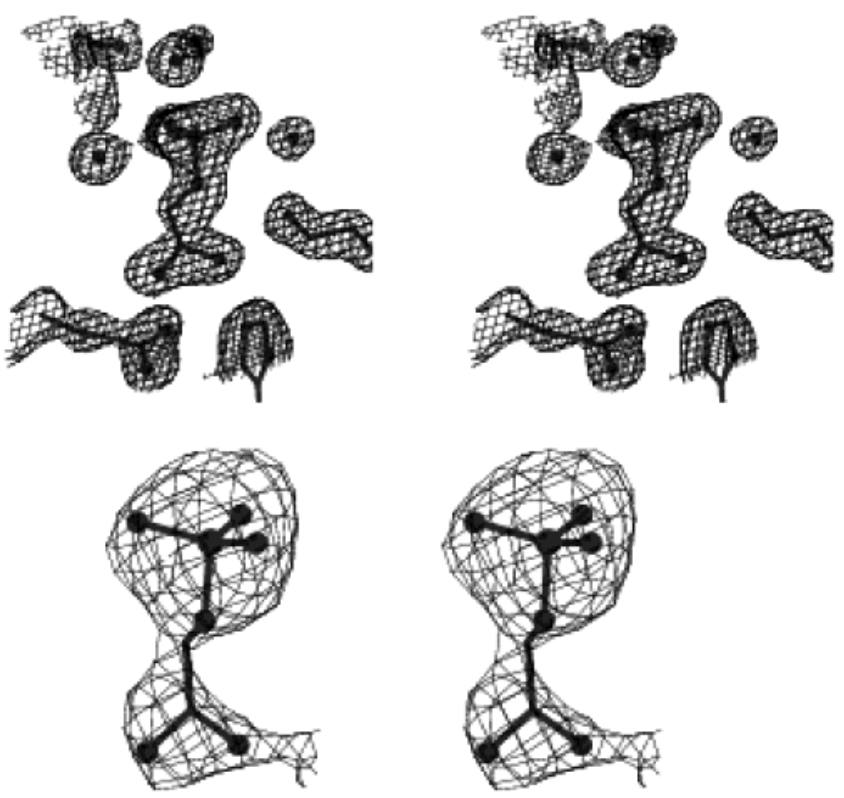

FIGURE 4: Map $\left(2 m F_{\mathrm{o}}-D F_{\mathrm{c}}\right)$ for the ligand phosphoglycolate, $\mathrm{PG}$, in monoclinic (top panel) and orthorhombic (bottom panel) forms. The top and bottom maps are contoured at $1.2 \sigma$ and $1.0 \sigma$, respectively.

Several hydrogen bonds, both direct and water-mediated, originating from two stretches of residues [Gly210-Ser211Val212 (the phosphate binding helix) and Leu230-Val231Gly232-Asn233-Ala234 (loop 7)] hold the phosphate group of PG. As many as six water molecules appear to hold the inhibitor in position at the ligand binding site. Residues Lys12, Ser211, Gly232, and Asn233 are involved in direct interaction with phosphate oxygens (Table 3 and Figure 5a). The interaction between the bridging oxygen O1P of PG and NZ of Lys12 (3.59 $\AA$ ) is important, as this is thought to be one of the determinants of the substrate specificity of TIM. The proximity of the side chain hydroxyl of Ser211 and O2P $(3.69 \AA)$ and a water-mediated intersubunit interaction involving W506 and the OH group of Ser73 (Ser73 coming $2.86 \AA$ from a symmetry-related molecule) are observed.

Orthorhombic Form (Loop Open). Of the four independent subunits present in the asymmetric unit, clear density corresponding to bound PG was observed in only the B and D subunits (Figure 4, bottom panel). In the A and C subunits, density corresponding to a phosphate group was observed but the remainder of the ligand atoms could not be clearly defined. Importantly, in all the four subunits, the catalytic loop 6 (residues 166-176) adopts the open conformation, in contrast to the $C 2$ form described above.

Figure $5 \mathrm{~b}$ shows the LIGPLOT representation of PG in the B subunit of the PfTIM-PG complex. Table 3 also lists all polar interactions between protein and ligand atoms using a distance cutoff of $3.8 \AA$ A. Residues Ser211 and Asn233 directly interact with the phosphate group of the ligand. In the B subunit, one of the phosphate oxygens (O3P) hydrogen bonds with both the main chain nitrogen $(2.99 \AA)$ and side chain OD1 (3.10 ̊) of Asn233. Another oxygen of the phosphate $(\mathrm{O} 4 \mathrm{P})$ hydrogen bonds with side chain OG of Ser211 (2.99 $\AA)$ and possibly with the main chain $\mathrm{N}$ of Val212 (3.80 ̊). O4P also hydrogen bonds with a water molecule (W580, $2.79 \AA$ ), which in turn interacts with peptide $\mathrm{N}$ of Val212. The other two oxygen atoms, O1P and $\mathrm{O} 2 \mathrm{P}$, form hydrogen bonds with two water molecules and 


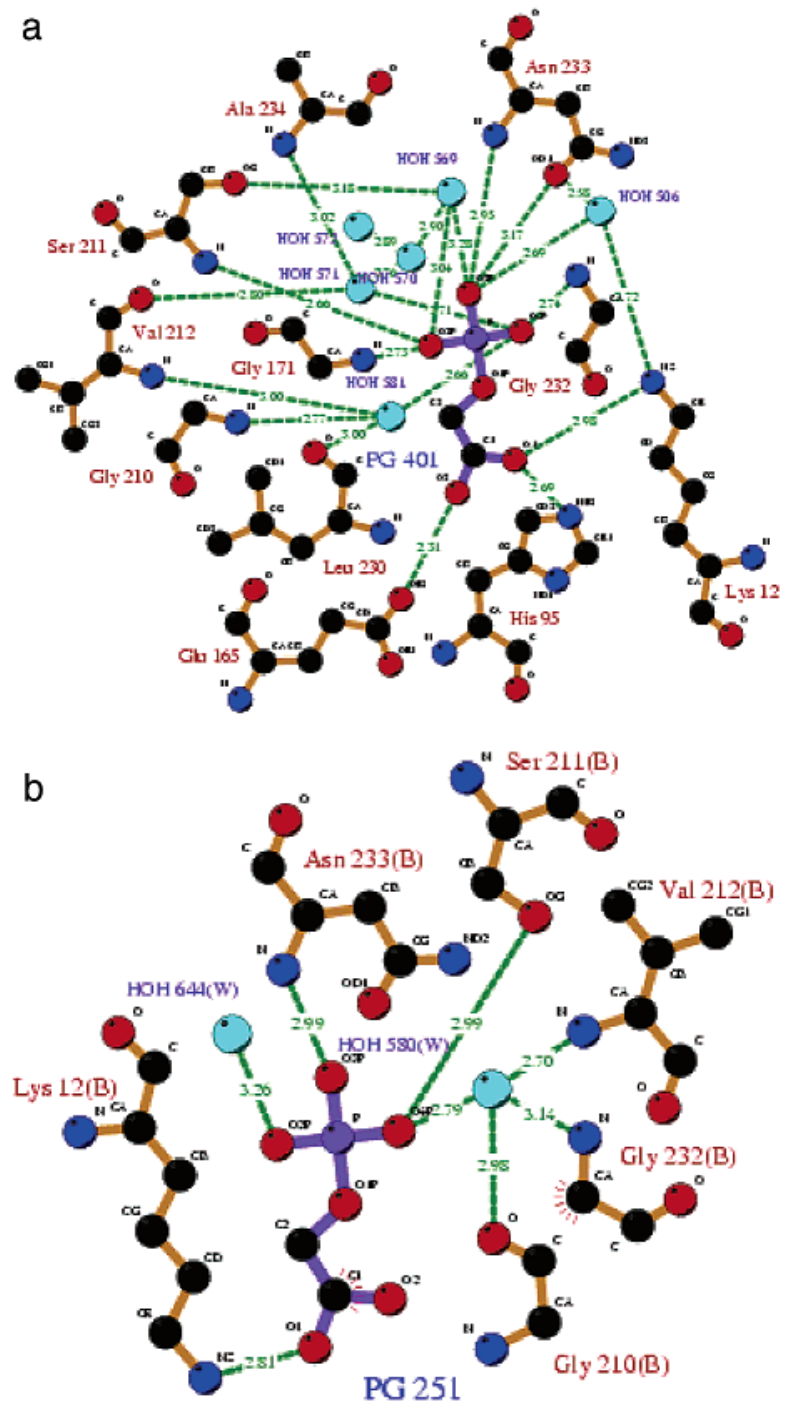

FIGURE 5: LIGPLOT representations for the bound phosphoglycolate, PG, in (a) monoclinic and (b) orthorhombic forms.

one water molecule, respectively. These water molecules in turn interact with Lys12 and Ser73 of the A subunit.

Comparable hydrogen bonding interactions with the phosphate group are also observed in the $\mathrm{D}$ subunit. The residues participating in direct interaction with phosphate oxygens are Gly232, Ser211, and Asn233. As in the B subunit, water molecules, W515 and W645, mediate the interaction between Lys12 and Ser73 of the neighboring C subunit. The phosphate group of the diverse ligands studied in TIM-inhibitor complexes provides an important site for anchoring the ligand at the enzyme active site. Comparison of the phosphate group environment in the $C 2$ form of the PfTIM-PG complex on one hand and the orthorhombic form of the PfTIM-PG complex on the other suggests that catalytic loop closure does result in a readjustment of the phosphate group at the binding site with extrusion of water molecules.

At the carboxylate end of $\mathrm{PG}$, the $\mathrm{O} 1$ atom hydrogen bonds with the amino group NZ of Lys12 in both the B and D subunits. In these subunits, the distances between $\mathrm{O} 1$ and NE2 of His95 are 3.73 and $3.96 \AA$, respectively. Interactions of the ligand (O1 atom) with the catalytic base Glu165 are weak and are mediated by water molecules W642 and W568.
Table 3: Distances between Protein and Ligand Atoms in the PfTIM-PG Complex in the B Subunit of the Orthorhombic Form and in the Active Site of the Monoclinic Form Using a Cutoff Distance of $3.8 \AA$

\begin{tabular}{|c|c|c|c|c|c|}
\hline \multicolumn{3}{|c|}{$P 2_{1} 2_{1} 2_{1}$ form (B subunit) } & \multicolumn{3}{|c|}{$C 2$ form } \\
\hline atom & atom & distance $(\AA)$ & atom & atom & distance $(\AA)$ \\
\hline \multirow[t]{3}{*}{$\mathrm{O} 1$} & Lys12B NZ & 2.81 & $\mathrm{O} 1$ & His95 NE2 & 2.69 \\
\hline & W644 O & 3.79 & & Glu165 OE2 & 3.47 \\
\hline & His95B NE2 & 3.73 & & Lys12 NZ & 2.98 \\
\hline \multirow[t]{2}{*}{$\mathrm{O} 2$} & Leu230B O & 3.49 & $\mathrm{O} 2$ & His95 NE2 & 3.58 \\
\hline & Gly232B N & 3.26 & & Glu165 OE2 & 2.31 \\
\hline \multirow[t]{2}{*}{ O1P } & W644 O & 3.69 & O1P & Gly $232 \mathrm{~N}$ & 3.79 \\
\hline & W580 O & 3.51 & & Lys12 NZ & 3.59 \\
\hline \multirow[t]{5}{*}{$\mathrm{O} 2 \mathrm{P}$} & W644 O & 3.26 & & Ala169 O & 3.64 \\
\hline & & & & Gly171 N & 2.73 \\
\hline & & & & Ser211 N & 2.66 \\
\hline & & & & Ser211 OG & 3.69 \\
\hline & & & & W569 O & 3.04 \\
\hline \multirow[t]{4}{*}{$\mathrm{O} 3 \mathrm{P}$} & Asn233B OD1 & 3.10 & $\mathrm{O} 3 \mathrm{P}$ & W569 O & 3.28 \\
\hline & Asn233B N & 2.99 & & W506 O & 2.69 \\
\hline & & & & Asn233 N & 2.95 \\
\hline & & & & Asn233 OD1 & 3.17 \\
\hline \multirow[t]{11}{*}{ O4P } & Ser211B OG & 2.99 & O4P & Ser211 N & 3.62 \\
\hline & Val212B N & 3.80 & & Gly232 N & 2.74 \\
\hline & W580 O & 2.79 & & W571 O & 2.71 \\
\hline & & & & W581 O & 2.66 \\
\hline & & & & Asn $233 \mathrm{~N}$ & 3.79 \\
\hline & & & $P$ & Gly171 N & 3.80 \\
\hline & & & & Ser211 N & 3.66 \\
\hline & & & & W569 O & 3.48 \\
\hline & & & & Gly $232 \mathrm{~N}$ & 3.69 \\
\hline & & & & W571 O & 3.68 \\
\hline & & & & W581 O & 3.71 \\
\hline
\end{tabular}

$\mathrm{O} 2$ of the ligand weakly hydrogen bonds with the main chain $\mathrm{O}$ of Leu230 and $\mathrm{N}$ of Gly232 (Table 3). In the B subunit, the main chain $\mathrm{N}$ of Gly232 interacts with $\mathrm{O} 2$, in contrast to the O of Gly209 found in the D subunit. Notably, none of the atoms of the flexible loop interacts with the ligand as the flexible loop is in the open conformation.

\section{Displacement of Phe96 upon Loop Closure}

A novel feature of the structures of the PfTIM-PG complexes is the observation of both the loop open and loop closed conformations in the ligand-bound state. Of the more than 30 structures of TIM-ligand complexes available in the Protein Data Bank, the overwhelming majority of the ligand-bound structures adopt the loop closed conformation. Indeed, the only example of an enzyme-inhibitor complex adopting the loop open conformation is the structure of TrypTIM bound to $N$-hydroxy-4-phosphonobutanamide [4PBH (63)]. In this case, the loop open conformation appears to be a consequence of crystal contacts. Both the loop open and partially closed forms of the trypanosomal enzyme have been observed in the crystals where sulfate ion occupies the ligand binding site (64).

In addition to the loop open, ligand-bound form of the PfTIM-PG complex, we have also obtained examples of the ligand-bound loop open state of PfTIM in complex with the inhibitors 3-phosphoglycerate (3PG) and glycerol 3-phosphate (G3P; 65). Thus, PfTIM appears to show a marked tendency toward adopting the loop open conformation even in the presence of bound ligands, in marked contrast to the behavior of TIMs from other sources. In searching for a specific cause for the stabilization of the loop open conformation, we assumed that Phe96 may act as a steric impedi- 

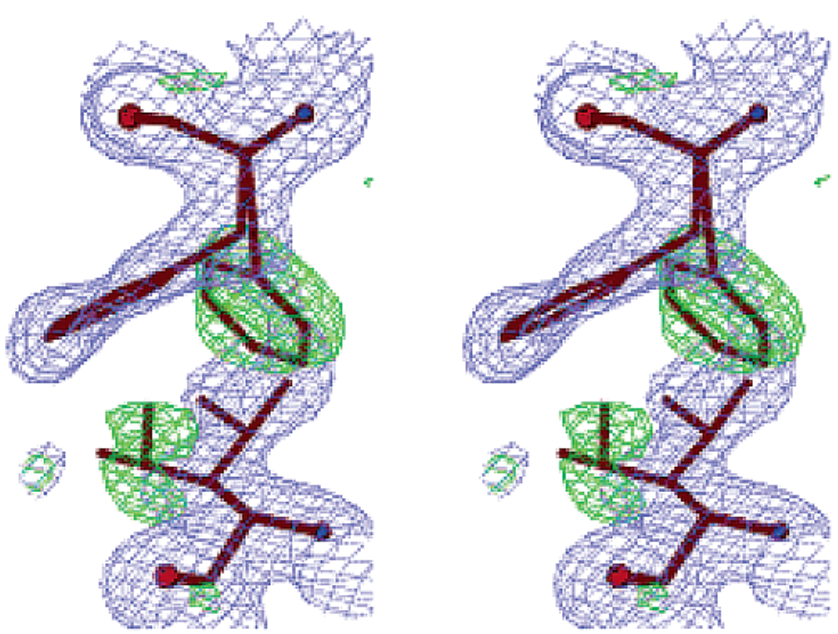

FIGURE 6: Electron density for alternate conformations of Phe96 and Leu167 in the PfTIM-PG complex, $C 2$ form. The $2 m F_{0}-$ $D F_{\mathrm{c}}$ (blue) and $m F_{\mathrm{o}}-D F_{\mathrm{c}}$ (green) maps are shown at $1.0 \sigma$ and $2.5 \sigma$, respectively. Blue and green contours correspond to major and minor conformations, respectively.

ment to loop closure. Modeling studies suggest that loop closure in PfTIM would place the Phe96 and Ile170 side chains in sterically unacceptable positions. The observation, in this study, of a loop closed form in the PfTIM-PG complex suggests that this mutation does not render loop closing impossible. Indeed, the unfavorable interaction between the phenyl ring of Phe96 and the side chain of Ile170 (a loop 6 residue) is avoided in the monoclinic form of the PfTIM-PG complex by a change in the orientation of the Phe96 side chain.

This reorientation, however, leads to a steric clash between Phe96 and Leu167 as positioned in the native unliganded structure (2), forcing the latter to adopt a new conformation. Careful inspection of the electron density maps suggests alternate conformations for both these residues. Figure 6 shows the electron density corresponding to these residues in the PfTIM-PG $C 2$ form. These alternate positions of Phe96 and Leu167 were refined to group occupancies of 80 and $20 \%$, respectively. These conformations are different from those found in the unbound (2) and ligand-bound, loop open forms of PfTIM. The $\chi^{1}$ angle of Phe96 in the latter forms is $\sim 95^{\circ}$ and is changed to $-166^{\circ}$ in the major conformation found in the $C 2$ form. Similarly, $\chi^{1}$ angles for Leu 167 are $-48^{\circ}$ and $-173^{\circ}$ in PfTIM and the PfTIM-PG complex, respectively. The $\chi^{1}$ angles for Phe96 and Leu167 in the minor conformation are $-128^{\circ}$ and $-173^{\circ}$, respectively. Thus, the Phe96 side chain displays substantial torsional freedom and occurs in a variety of conformations: trans (major conformation in the PG C2 form), gauche ${ }^{-}$ (minor conformation in the PG C2 form), and gauche (in PfTIM and its complexes with open loop structures).

\section{Features of Catalytic Loop Open and Closed Conformations of PfTIM}

The availability of the structures of PfTIM in the unliganded form and liganded complexes, with both loop open and loop closed conformations, provides an opportunity to examine the local structural changes that occur as the loop closes over the active site. We have chosen the following three structures for comparison: (1) unbound PfTIM, loop open [2.2 A resolution (2)]; (2) PfTIM-3PG complex, ligand-bound, loop open $[2.4 \AA$ resolution (65), PDB entry 1M7O]; and (3) PfTIM-PG complex $C 2$ form, ligand-bound, loop closed [1.9 А resolution (this paper)].

For the purpose of comparison of the ligand-bound, loop open form, we have chosen the PfTIM-3PG complex over the PfTIM-PG complex, $P 2_{1} 2_{1} 2_{1}$ form described in this paper, because of higher crystallographic resolution. The details of the two structures are similar.

Superposition of the active site (using $\mathrm{C}^{\alpha}$ atoms of residues Lys12, His95, Glu165, and residues adjacent to these) revealed only minor differences in the position of the side chains of the active site residues. Major differences are limited to the water molecules at the active site (Figure 7). A comparison of the unliganded and loop open complex structure (PfTIM-3PG) reveals that of the water molecules, W536 and W589 bound to His95 and Glu165, respectively, in the former, only W536 is expelled from the active site on ligand binding. However, in the case of the bound closed conformation (PfTIM-PG C2 form), both of these water molecules are expelled upon loop closure, resulting in direct interaction between the catalytic base and the ligand, PG. The $\chi^{1}, \chi^{2}$, and $\chi^{3}$ angles of Glu 165 are $-51^{\circ},-173^{\circ}$, and $-79^{\circ} ;-55^{\circ}, 177^{\circ}$, and $-66^{\circ}$; and $-89^{\circ},-167^{\circ}$, and $-40^{\circ}$, in unliganded, bound open, and bound closed forms of PfTIM, respectively. Interestingly, changes in torsion angles do not seem to affect the interaction between the main chain $\mathrm{N}$ of Phe96 and OE1 of Glu165. The distances between OE1 of Glu165 and peptide N of Phe96 in these forms (3.04, 3.10, and $3.10 \AA$, respectively) are nearly the same. This is in contrast to yeast and chicken TIM complexes where the interaction between the main chain $\mathrm{N}$ and $\mathrm{OE} 1$ of the catalytic base breaks [corresponding distance is $5.89 \AA$ in the yeast TIM-PG complex (55)], resulting in movement of the catalytic base toward the ligand. Two other water molecules (W590 and W653) of unliganded enzymes become displaced on ligand binding. In both bound open and bound closed forms, phosphate oxygens seem to occupy equivalent positions. The displaced water molecules, however, mediate the protein-ligand interactions in both forms. Another notable common feature in all the three states is the watermediated link between Lys12 and Ser73 (from the neighboring subunit). Water molecule W650 is placed 3.37 and 3.11 $\AA$ from NZ of Lys12 and OH of Ser73, respectively, in unbound PfTIM. The corresponding distances involving W578 in the bound open state are 2.69 and $2.56 \AA$, respectively. These distances between W506 in the PfTIMPG $C 2$ complex are 2.86 and $2.72 \AA$, respectively. The shortening of these distances suggests modulation of intersubunit interactions upon ligand binding. In the PfTIM3PG complex, the catalytic loop (loop 6) is involved only in water-mediated interaction with the bound ligand and remains in the open conformation (65). This observation taken into consideration with the minor changes of the catalytic residues, as described above, suggests that triosephosphate isomerase presents a "preformed" binding site. In the case of the bound closed conformation, as observed in the PfTIM-PG complex ( $C 2$ form), the loop closes over the active site, leading to further extrusion of water molecules from the active site. Drying out the active site may, in fact, be a process which facilitates catalysis. 

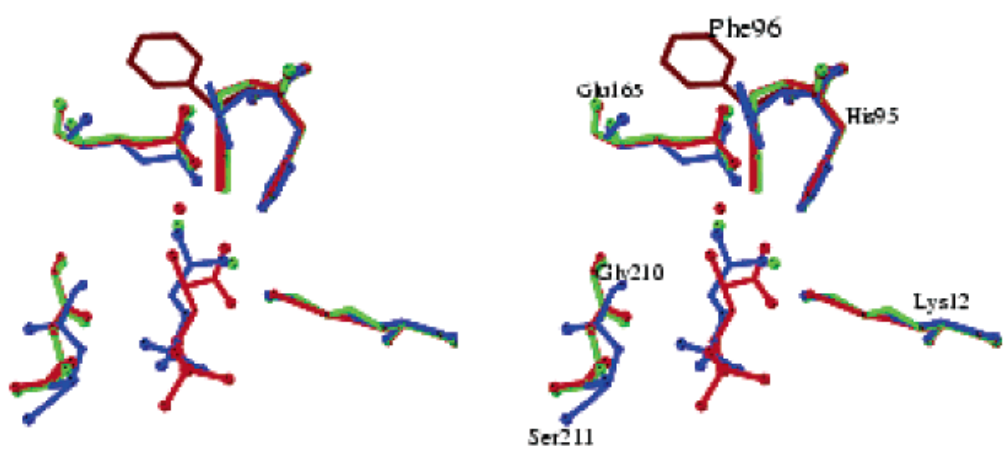

FIGURE 7: Active site superposition of unbound (green), bound open (3PG complex, red), and bound closed (PG complex, $C 2$ form, blue) forms of PfTIM.
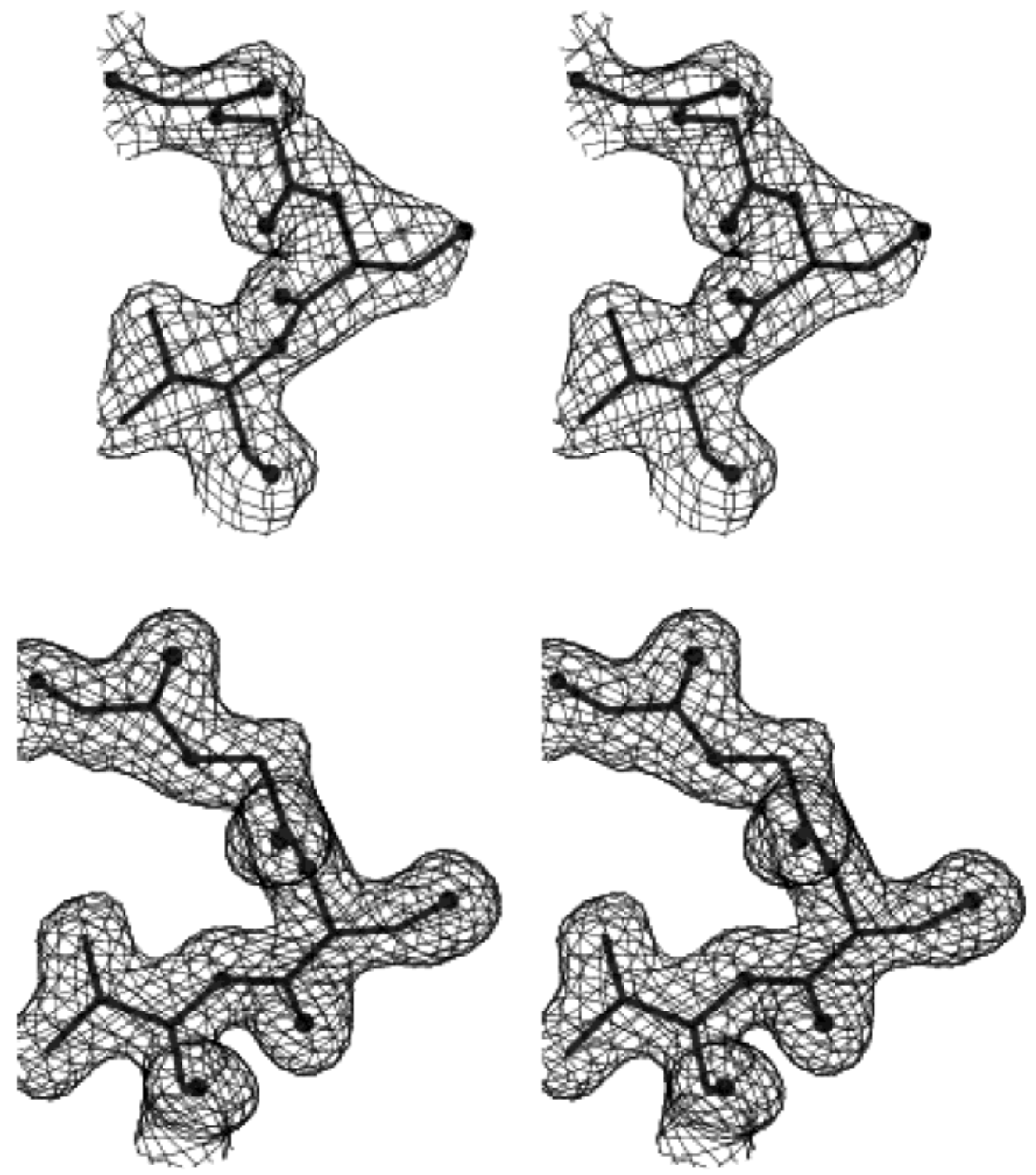

FIGURE 8: Electron density, $2 m F_{\mathrm{o}}-D F_{\mathrm{c}}$ map contoured at 1.0 $\sigma$, for the Gly209-Gly210-Ser211-Val212 segment, in bound open (3PG complex, top panel) and bound closed (PG complex, $C 2$ form, bottom panel) forms of PfTIM. The peptide flip about the Gly210-Ser211 bond occurs upon loop closure in the PfTIM-PG complex.

Loop closure in TIM on ligand binding is accompanied by a flip about the Gly210-Ser211 (PfTIM numbering) peptide bond. Figure 8 shows the electron density in this region for both PfTIM-3PG and PfTIM-PG (C2 form) complexes. The $\phi$ and $\varphi$ values of these residues in unbound PfTIM, 3PG-bound PfTIM, and the $C 2$ form of the PGPfTIM complex are $-87^{\circ}$ and $-162^{\circ},-104^{\circ}$ and $-166^{\circ}$, and $137^{\circ}$ and $-90^{\circ}$ for Gly 210 , respectively, and $-70^{\circ}$ and $110^{\circ},-76^{\circ}$ and $112^{\circ}$, and $59^{\circ}$ and $39^{\circ}$ for Ser 211 , respectively. The peptide flip which accompanies loop closure results in large changes in the Ramachandran angles for the Gly210-Ser211 peptide bond, with the later adopting a positive $\phi$ value which is energetically unfavorable for L-residues (66). Gly210 adopts $\phi$ and $\varphi$ values which are permissible only for this residue. Notably, the segment of residues 208-212 is completely conserved in all TIM sequences (Figure 1). It is conceivable that even a conservative substitution of Ser211 with Thr, a $\beta$-branched residue, may alter the catalytic properties of the enzyme. It has been suggested that the energy requirement for this peptide flip is compensated by the binding energy and that the loop closure and peptide flip occur concurrently.

\section{Enzyme Inhibition by Phosphoglycolate and 3-Phosphoglycerate}

The structural investigations described in this paper and the earlier report (65) provide a detailed view of the binding of the inhibitors phosphoglycolate and 3-phosphoglycerate 
Table 4: Inhibition Constants $\left(K_{\mathrm{i}}, \mathrm{mM}\right)$ in Millimolar

Concentrations of Phosphoglycolate and 3-Phosphoglycerate for Triosephosphate Isomerases

\begin{tabular}{lcl}
\hline \multicolumn{1}{c}{ organism } & phosphoglycolate & 3-phosphoglycerate (isomer; ref) \\
\hline$P$. falciparum & $0.029 \pm 0.009$ & $1.9 \pm 0.5$ (D-isomer; this paper) \\
T. brucei & $0.027 \pm 0.005$ & $1.3 \pm 0.4$ (D,L-mixture; 67) \\
rabbit muscle & $0.014 \pm 0.003$ & $0.51 \pm 0.07$ (D,L-mixture; 67) \\
yeast & $0.030 \pm 0.006$ & $1.1 \pm 0.1$ (D,L-mixture; 67) \\
\hline
\end{tabular}

to PfTIM. To attempt to draw a correlation between the observed structures and the efficiency of inhibition, we have determined the inhibition constants $\left(K_{\mathrm{i}}\right)$ for the binding of the two ligands to the malarial enzyme. The results are summarized in Table 4. The data for triosephosphate isomerases from other sources (67) are also listed for comparison. The inhibition constants determined for phosphoglycolate are very similar to those observed for the yeast, rabbit, and trypanosomal enzymes. Interestingly, 3PG appears to be a significantly poorer inhibitor of PfTIM as compared to the enzyme from other sources (Table 4). It should be noted that the $K_{\mathrm{i}}$ values in earlier studies correspond to the racemic ligand D,L-3-phosphoglycerate, while in the study presented here, we have used only the D-isomer. Previous reports have established that the L-forms are much weaker inhibitors of TIMs (65). It would thus appear, as a crude approximation, that the 3-phosphoglycerate is $\sim 3$-fold less effective as an inhibitor of the Plasmodium enzyme than the trypanosomal enzyme. Inspection of the structure of the PfTIM-3PG complex reveals that the hydroxyl group of the $\mathrm{C} 2$ atom of the inhibitor makes close contact with the CD1 and CE1 atoms of the aromatic ring of the Phe96 side chain (65). The observed $\mathrm{O}-\mathrm{C}$ distances are 4.00 and $3.48 \AA$, respectively. In TrypTIM, Phe96 is replaced with Ser. It is likely that the S96F mutation in the Plasmodium enzyme results in less effective binding of 3-phosphoglycerate.

\section{CONCLUSION}

The availability of structures at reasonably high resolution for unliganded PfTIM and the ligand-bound form of the enzyme in both the loop open and loop closed conformations provides a wealth of structural detail about the movements of critical residues and strongly bound water molecules as the active site undergoes large conformational changes. The observation of both loop open and closed forms in the crystals of PfTIM-ligand complexes supports the view that the transition between the loop open and loop closed forms may be significantly influenced by ligand binding $(25,26)$. NMR studies suggest that the conformational transition between the open and closed states of yeast TIM occurs with a rate of $10^{4} \mathrm{~s}^{-1}$ (27). Substrate binding to the open form of TIM is clearly the first step in moving toward the catalytically competent state, which is undoubtedly the loop closed form (23). The preponderance of loop open conformations in the case of PfTIM-ligand complexes suggests that mutational changes may indeed affect the stability of the open and closed states and could considerably alter the dynamics of interconversion, by influencing the activation barriers. The important mutational change in PfTIM at residue 96, where the parasite enzyme has Phe whereas enzymes from all other sources have Ser, may account for the unusual ligand-bound structures.
The determination of the structure of the PfTIM-ligand complex reported in this paper together with structures of complexes with 3PG and G3P clearly establishes that loop closure does not necessarily have to accompany ligand binding. This is consistent with the earlier study by Sampson and Knowles (28), who showed that loop closure occurs after the formation of the Michaelis complex. The elegant NMR studies of McDermott and co-workers (25) have also demonstrated that in solution TIM exists in a dynamic equilibrium between the loop open and loop closed forms. The results reported in this paper provide a snapshot of both states with the ligand occupying the active site. Novel properties of $P$. falciparum TIM are likely to derive from the Phe residue at position 96, making this enzyme an attractive system for future investigations of loop dynamics in solution.

Clearly, even in an enzyme that has been studied as well as TIM, we are yet to reach a stage where a detailed view of the molecular events during catalysis is obtained. While specific interactions undoubtedly guide the relatively small substrates to the binding site, catalysis requires some largescale motions like that of the catalytic loop (loop 6) and some subtle motions like movement of the active site base, Glu165, and the flip of the Gly210-Ser211 peptide bond. The wealth of structural detail accumulating from the studies of TIMinhibitor complexes should serve to catalyze further mutational analysis of the dynamic processes, which are undoubtedly critical for the activity of the enzyme.

\section{REFERENCES}

1. Subbayya, I. N. S., Ray, S. S., Balaram, P., and Balaram, H. (1997) Indian J. Med. Res. 106, 79-94.

2. Velanker, S. S., Ray, S. S., Gokhale, R. S., Suma, S., Balaram, H., Balaram, P., and Murthy, M. R. N. (1997) Structure 5, 751761.

3. Roth, E., Jr., Kuvlin, V., Miwa, S., Yoshida, A., Akatusuka, J., Cohen, S., and Rosa, R. (1988) Blood 71, 1408-1413.

4. Maes, D., Zeelan, J. P., Thank, N., Beaucamp, N., Alvarez, M., Thi, M. H. D., Backmann, J., Martial, J. A., Wyns, L., Jaenicke, R., and Wierenga, R. K. (1999) Proteins: Struct., Funct., Genet. 37, 441-453.

5. Delboni, L. F., Mande, S. C., Delrue, F. R., Mainfroid, V., Turley, S., Vellieux, F. M. D., Martial, J. A., and Hol, W. G. J. (1995) Protein Sci. 4, 2594-2604.

6. Noble, M. E. M., Zeelan, J. P., Wierenga, R. K., Mainfroid, V., Goraj, K., Gohimont, A. C., and Martial, J. A. (1993) Acta Crystallogr. D49, 403-417.

7. Alvarez, M., Zeelan, J. P., Mainfroid, V., Delrue, F. R., Martial, J. A., Wyns, L., Wierenga, R. K., and Maes, D. (1998) J. Biol. Chem. 273, 2199-2206.

8. Zhang, Z., Sugio, S., Komives, E. A., Liu, K. D., Knowles, J. R., Petsko, G. A., and Ringe, D. (1994) Biochemistry 33, 2830-2837.

9. Mande, S. C., Mainfroid, V., Kalk, K. H., Goraj, K., Martial, J. A., and Hol, W. G. J. (1994) Protein Sci. 3, 810-821.

10. Lolis, E., Alber, T., Davenport, R. C., Rose, D., Hartman, F. C., and Petsko, G. (1996) Biochemistry 29, 6609-6618.

11. Noble, M. E. M., Wierenga, R. K., Lambeir, A. M., Opperdoes, F. R., Thunnissen, A. M. W. H., Kalk, K. H., Groendijk, H., and Hol, W. G. J. (1991) Proteins: Struct., Funct., Genet. 10, 5069.

12. Williams, J. C., Zeelan, J. P., Neubauer, G., Vriend, G., Backmann, J., Michels, A. M., Lambier, A., and Wierenga, R. K. (1999) Protein Eng. 12, 243-250.

13. Alber, T. C., Davenport, R. C., Jr., Giammona, D. A., Lolis, E., Petsko, G. A., and Ringe, D. (1987) Cold Spring Harbor Symp. Quant. Biol. 52, 603-613.

14. Hermes, J. D., Blacklow, S. C., and Knowles, J. R. (1990) Biochemistry 29, 4099-4108. 
15. Komives, E. A., Lougheed, J. C., Liu, K., Suigio, S., Zhang, Z., Petsko, G. A., and Ringe, D. (1995) Biochemistry 34, 1361213621.

16. Komives, E. A., Lougheed, J. C., Liu, K., Zhang, Z., Suigio, S., Narayana, N., Xuong, H. N., Petsko, G. A., and Ringe, D. (1995) Biochemistry 34, 15474-15484.

17. Zhang, Z., Komives, E. A., Sugio, S., Blacklow, S. C., Narayana, N., Xuong, H. N., Stock, A. M., Petsko, G. A., and Ringe, D. (1999) Biochemistry 38, 4389-4397.

18. Singh, S. K., Kapil, M., Balaram, H., and Balaram, P. (2001) FEBS Lett. 501, 19-23.

19. Knowles, J. R., and Albery, W. J. (1977) Acc. Chem. Res. 10, 105-111.

20. Knowles, J. R. (1991) Nature 350, 121-124.

21. Alber, T., Banner, D. W., Bloomer, A. C., Petsko, G. A., Philips, D., Rivers, P. S., and Wilson, I. A. (1981) Philos. Trans. R. Soc. London, Ser. B 293, 159-171.

22. Wierenga, R. K., Borchert, T. V., and Noble, M. E. M. (1992) FEBS Lett. 1, 34-39.

23. Pompliano, D. L., Peyman, A., and Knowles, J. R. (1990) Biochemistry 29, 3186-3194.

24. Joseph, D., Petsko, G. A., and Karplus, M. (1991) Science 249, $1425-1428$

25. Williams, J. C., and McDermott, A. E (1995) Biochemistry 34, 8309-8319.

26. Rozovsky, S., Jogl, G., Tong, L., and McDermott, A. E. (2001) J. Mol. Biol. 310, 271-280.

27. Rozovsky, S., and McDermott, A. E. (2001) J. Mol. Biol. 310, 281.

28. Sampson, N. S., and Knowles, J. R. (1992) Biochemistry 31, 8482-8487.

29. Sun, J., and Sampson, N. S. (1999) Biochemistry 38, $11474-$ 11481.

30. Xiang, J., Jeonghoon, S., and Sampson, N. S. (2001) J. Mol. Biol. 307, 1103-1112.

31. Ranie, J., Kumar, V. P., and Balaram, H. (1993) Mol. Biochem. Parasitol. 61, 159-170.

32. Otwinowski, Z., and Minor, W. (1997) Methods Enzymol. 276, 307-325.

33. Diederichs, K., and Karplus, M. (1997) Nat. Struct. Biol. 4, 269275.

34. Velankar, S. S. (1997) Structural studies on thymidylate synthase and triosephosphate isomerase, Ph.D. Thesis, Indian Institute of Science, Bangalore, India.

35. Rossmann, M. G. (1990) Acta Crystallogr. A46, 73-82.

36. Navaza, J., and Saludian, P. (1997) Methods Enzymol. 276, 581593.

37. Pannu, N. S., and Read, R. J. (1996) Acta Crystallogr. A52, 659668.

38. Brunger, A. T., Adams, P. D., Clore, G. M., Delano, W. L., Gros, P., Grosse-Kunstleve, R. W., Jiang, J. S., Kuszewski, J., Nilges, M., Pannu, N. S., Read, R. J., Rice, L. M., Simonson, T., and Warren, G. L. (1998) Acta Crystallogr. D54, 905-921.

39. Brunger, A. T. (1992) Nature 355, 472-475.
40. Kleywegt, G. J., and Brunger, A. T. (1996) Structure 4, 897904.

41. Read, R. J. (1986) Acta Crystallogr. A42, 140-149.

42. Jones, T. A. (1978) J. Appl. Crystallogr. 11, 268-272.

43. Jones, T. A., Zou, J. Y., Cowan, S. W., and Kjeldgaard, M. (1991) Acta Crystallogr. A47, 110-119.

44. Cohen, G. E. (1997) J. Appl. Crystallogr. 30, 1160-1161.

45. Wallace, A. C., Laskowski, R. A., and Thornton, J. M. (1995) Protein Eng. 8, 127-134.

46. Collaborative Computational Project Number 4 (1994) Acta Crystallogr. D50, 760-763.

47. Kleywegt, G. J., and Jones, T. A. (1996) Acta Crystallogr. D52, 829-832.

48. Laskowski, R. A., MacArthur, M. W., Moss, D. S., and Thornton, J. M. (1993) J. Appl. Crystallogr. 26, 283-291.

49. Vriend, G. (1990) J. Mol. Graphics 8, 52-56.

50. Kraulis, P. J. (1991) J. Appl. Crystallogr. 4, 946-950.

51. Esnouf, R. M. (1999) Acta Crystallogr. D55, 938-940.

52. Merrit, E. A., and Bacon, D. J. (1997) Methods Enzymol. 277, 505-524.

53. InsightII, Accelrys, San Diego.

54. Plaut, B., and Knowles, J. (1972) Biochem. J. 129, 311-320.

55. Lolis, E., and Petsko, G. A. (1990) Biochemistry 29, 6619-6625.

56. Alvarez, M., Wouters, J., Maes, D., Mainfroid, J. A., Rentier, F. D., Wyns, L., Depiereux, E., and Martial, J. A. (1999) J. Biol. Chem. 274, 19181-19187.

57. Borchert, T. V., Radhakishan, K. V., Zeelen, J. Ph., Schliebs, W., Thanki, N., Abagyan, R., Jaenicke, R., and Wierenga, R. K. (1995) Structure 5, 669-679.

58. Thanki, N., Zeelan, J. Ph., Mathieu, M., Jaenicke, R., Abagyan, R. A., Wierenga, R. K., and Schliebs, W. (1997) Protein Eng. 10, 159-167.

59. Ramakrishnan, C., and Ramachandran, G. N. (1965) Biophys. J. 5, 909-933.

60. Kleywegt, G. J., and Jones, A. T. (1996) Structure 4, 1395-1400.

61. Noble, M. E. M., Virlinde, C. L. M. J., Groendijk, H., Kalk, K. H., Wierenga, R. K., and Hol, W. G. J. (1991) J. Med. Chem. 34, 2709-2718.

62. Zhang, Z., Sugio, S., Komives, E. A., Liu, K. D., Knowles, J. R., Petsko, G. A., and Ringe, D. (1994) Biochemistry 33, 2380-2387.

63. Verlinde, C. L. M. J., Witmans, C. J., Pijning, T., Kalk, K. H., Hol, W. G. J., Callens, M., and Opperdoes, F. R. (1992) Protein Sci. 1, 1578-1584.

64. Wierenga, R. K., Noble, M. E. M., Vriend, G., Nauche, S., and Hol, W. G. J. (1991) J. Mol. Biol. 220, 995-1015.

65. Parthasarathy, S., Balaram, H., Balaram, P., and Murthy, M. R. N. (2002) Acta Crystallogr. D59 (in press).

66. Stites, W. E., Meeker, A., and Shortle, D. (1994) J. Mol. Biol. 235, 27-32.

67. Lambeir, A. M., Opperdoes, F. R., and Wierenga, R. K. (1987) Eur. J. Biochem. 168, 69-74.

$\mathrm{BI} 025783 \mathrm{~A}$ 